\title{
Influencia de agentes crioprotectores sobre las cinéticas de congelación de lechuga (Lactuca sativa L.)
}

\section{Influence of cryoprotective agents on lettuce freezing kinetics (Lactuca sativa L.)}

\author{
Natalia María Herrera-García; Ingrith Johana López-Guerrero²; Liliana Serna-Cock
}

\begin{abstract}
'Estudiante Ingeniería Agroindustrial. Universidad Nacional de Colombia. Palmira, Valle del Cauca, Colombia; e-mail: nmherrerag@unal.edu.co, (Dhttp://orcid.org/0000-0003-3000-7732

${ }^{2}$ Estudiante Ingeniera Agroindustrial. Universidad Nacional de Colombia. Palmira, Valle del Cauca, Colombia; e-mail: ijlopezg@unal.edu.co, (Dhttp://orcid.org/0000-0002-7849-0826

${ }^{3}$ Bacterióloga, Ph.D. Universidad Nacional de Colombia, Facultad de Ingeniería y Administración. Palmira, Valle del Cauca, Colombia. e-mail: Iserna@unal. edu.co, Dhttp://orcid.org/0000-0003-2911-0871
\end{abstract}

*autor de Correspondencia: Iserna@unal.edu.co

Cómo citar: Herrera-García, N.M.; López-Guerrero, I.J.; Serna-Cock, L. 2019. Influencia de agentes crioprotectores sobre las cinéticas de congelación de lechuga (Lactuca sativa L.). Rev. U.D.C.A Act. \& Div. Cient. 22(2):e1305. http://doi.org/10.31910/rudca.v22.n2.2019.1305

Artículo de acceso abierto publicado por Revista U.D.C.A Actualidad \& Divulgación Científica, bajo una licencia Creative Commons CC BY-NC 4.0

Recibido: Junio 19 de 2018

Aceptado: Julio 21 de 2019

Editado por: Ingeborg Zenner de Polanía

\section{RESUMEN}

La lechuga es una hortaliza de hoja que no resiste la congelación. Como una alternativa de conservación de lechuga Lactuca sativa L. "Lollo Bionda", se evaluó el efecto de la aplicación de crioprotectores sobre cada una de las etapas de congelación de lechuga. En cada etapa, se evaluó el tiempo de duración, la velocidad y la temperatura de congelación. Como crioprotectores, se utilizaron soluciones de Aloe (Aloe barbadensis Miller), a concentraciones de 70, 80 y 90\%; almidón, a concentraciones de 0,5, 1 y $2 \%$ p/p y aceite de oliva. Las muestras, se impregnaron con los diferentes crioprotectores y se ultracongelaron. Las temperaturas del material vegetal, se registraron durante una hora, con intervalos de 5s; se construyeron las cinéticas de congelación y sobre las cinéticas, se identificaron las diferentes etapas de congelación. Se encontró que el tipo y la concentración del crioprotector altera, de manera estadísticamente significativa, la temperatura, el tiempo y la velocidad de cada una de las etapas de congelación. Las mayores concentraciones de solutos presentes en Aloe vera al 90\%, almidón al 2\% y aceite de oliva influyeron considerablemente en la acción crioprotectora de la lechuga.

Palabras clave: Aloe; almidón; crioprotectores; congelación; aceite de oliva.

\section{ABSTRACT}

Lettuce is a leafy vegetable that does not resist freezing. As an alternative to the lettuce Lactuca sativa L. "Lollo Bionda" lettuce conservation, the effect of the application of cryoprotectants on each of the freezing stages of lettuce was evaluated. In each stage, duration time, speed and freezing temperature were evaluated. Aloe (Aloe barbadensis Miller) solutions at concentrations of 70,80 and 
$90 \%$, starch at concentrations of $0.5,1$ and $2 \% \mathrm{w} / \mathrm{w}$ and olive oil were used as cryoprotectants. The samples were impregnated with the different cryoprotectants and deep-frozen. The temperatures of the plant material were recorded for one hour at intervals of 5 $\mathrm{s}$, the freezing kinetics were built and, on the kinetics, the different stages of freezing were identified. It was found that the type and concentration of the cryoprotectant alters in a statistically significant way the temperature, time and speed of each of the freezing stages. The highest concentrations of solutes present in $90 \%$ Aloe vera, $2 \%$ starch and olive oil significantly influenced the cryoprotective action of lettuce.

Keywords: Aloe; starch; cryoprotectants; freezing; olive oil.

\section{INTRODUCCIÓN}

La lechuga (Lactuca sativa L.) es uno de los vegetales de hoja con más consumo mundial, según la USDA (2016); para el 2015, se consumieron alrededor de $11,11 \mathrm{~kg}$ de lechuga por persona, de los cuales, el 55\% fue lechuga crespa verde. En Colombia, en el 2017, se produjeron 51,000t, con un crecimiento del 7\% (Ministerio de Agricultura y Desarrollo Rural, 2017). La lechuga es un producto perecedero, cuya vida de anaquel, dependiendo de la variedad, es de tan solo 2 semanas, cuando se almacena a temperaturas cercanas a los $0^{\circ} \mathrm{C}$ (Esparza et al. 2013). A esta rápida perecibilidad, se le suma el problema de las pérdidas poscosecha. En Colombia, se desperdiciaron en la postcosecha 1,4 millones de toneladas de frutas y verduras, cifra que incluye a la lechuga; de acuerdo con el Ministerio de Agricultura y Desarrollo Rural, el desperdicio en el 2015, correspondió a 58\% de la producción (MADR, 2017).

El almacenamiento refrigerado disminuye las pérdidas postcosecha de la mayoría de las frutas y verduras (Fabbri et al. 2018); sin embargo, deshidrata fácilmente los vegetales de hoja, como la lechuga.

La crioconservación es por excelencia el método de conservación de tejido animal y vegetal. Para tejidos vegetales, tradicionalmente, los protocolos de crioconservación se optimizaban para manzana, utilizando diversos métodos, como la vitrificación, la encapsulacióndeshidratación y la congelación lenta; los resultados de estas investigaciones permitieron utilizar estos métodos en papa y en otros productos vegetales (Kushnarenko et al. 2015). Las dificultades de la crioconservación están asociadas con las peculiaridades de las células vegetales, las cuales, tienen un gran tamaño, vacuolas grandes y alto contenido de agua (Engelmann, 2011). La muerte celular puede ocurrir, debido a la formación de hielo durante el proceso de congelación. Los cristales de hielo intracelular causan daños mecánicos durante la congelación, lo que resulta en la deshidratación celular (Romadanova et al. 2017). Los daños, se pueden evitar, a través de la eliminación del exceso de agua del producto o del uso de crioprotectores (Gurruchaga et al. 2015). Ambos enfoques tienen el mismo objetivo, de reducir o prevenir la formación de hielo en los tejidos (Romadanova et al. 2017).

La crioprotección consiste en la adición previa a la congelación, de una sustancia que es capaz de incorporarse a las células del tejido o a los espacios intracelulares, protegiendo la textura del material vegetal del daño causado por los cristales de hielo (Gurruchaga et al. 2018). Los crioprotectores son sustancias penetrantes o no penetrantes, de diferentes pesos moleculares, de baja toxicidad, que disminuyen el punto de congelación de una solución; con la aplicación de un crioprotector, las células estarán más deshidratadas y el gradiente osmótico al que estarán sometidas será menor (Pérez et al. 2016). Los movimientos de agua y de crioprotectores, a través de la membrana celular durante la crioprotección, se rigen por diversos parámetros, que son propios de cada tipo de célula a diferentes temperaturas. Estos parámetros son: el volumen osmóticamente inactivo, que se define como el agua que nunca dejará el interior celular, en respuesta a un aumento de concentración de solutos en el espacio extracelular, por estar asociado a las macromoléculas y estructuras intracelulares; la permeabilidad de la membrana celular al agua, a criopreservantes y a solutos y, el volumen de agua que ha atravesado la membrana, basado en que durante la deshidratación de la célula, sin congelación intracelular, se aumenta la concentración de electrolitos, sustratos, cofactores, proteínas celulares y, asimismo, se aumenta el transporte de agua al medio externo en congelación (Ávila-Portillo et al. 2006).

La crioprotección reabrió la posibilidad no solo de hacer más eficaz el procesado de congelación en la industria alimentaria, sino también, en otros sectores productivos (Năstase et al. 2016).

Grandes progresos se lograron con el desarrollo de técnicas de crioprotección, identificándose este campo como un área de prioridad para la investigación, desarrollo y transferencias de tecnología. Se han llevado a cabo investigaciones que evalúan la capacidad de protección de células vegetales y animales de diferentes criopotectores (Marco \& Serrano, 2012; Gurruchaga et al. 2018). En tejido animal, se ha reportado el efecto crioprotector de oligopéptidos obtenidos de hidrolizado de merluza y de colágeno, con aplicaciones en la crioprotección de pescado y en la protección a la desnaturalización de la proteína de Surimi. El crioprotector de naturaleza peptídica inhibe el desplazamiento de las moléculas de agua durante la congelación y estabiliza el agua en la grasa de algas, lo que demuestra el efecto crioprotector de estos péptidos (Gokoglu, 2019); sin embargo, estas tecnologías aplicadas a la conservación de frutas y de hortalizas pueden afectar la textura y otras propiedades sensoriales (Gómez-Sánchez et al. 2007).

La efectividad del criopotector dependerá de variables, como permeabilidad celular, volumen, relación superficie/área de la célula, la cual, es variable de acuerdo con la especie, tipo y estadio de la célula a congelar (Ávila-Portillo et al. 2006). El objetivo de esta investigación fue determinar la influencia de la aplicación de agentes crioprotectores en lechuga Lactuca sativa L. "Lollo Bionda" sobre la velocidad, la duración y la temperatura de las diferentes etapas de congelación.

\section{MATERIALES Y MÉTODOS}

Se utilizaron lechugas frescas L. sativa "Lollo Bionda" con humedad promedio de 95,01 $\pm 1,2$, provenientes de un mercado local del municipio de Palmira (Valle del Cauca, Colombia). Las lechugas, 
se seleccionaron teniendo en cuenta los requisitos mínimos de calidad, expuestos en la NTC 1064 (Icontec, 1994). Se utilizaron hojas enteras, sanas, de apariencia fresca, libres de daños causado por insectos, sin indicios de deshidratación. Las hojas, se lavaron por inmersión, en una solución acuosa de hipoclorito de sodio, a $150 \mathrm{ppm}, \mathrm{a} \pm 4^{\circ} \mathrm{C}$.

\section{Preparación de crioprotectores}

Gel de Aloe vera (Aloe barbadensis Miller): El gel fue extraído de hojas de Aloe vera provenientes de un cultivo del municipio de la Cumbre (Valle del Cauca, Colombia), las cuales, se desinfectaron y después, se filetearon manualmente, siguiendo el método propuesto por Domínguez et al. (2012). Se extrajo el gel manualmente y se homogenizó por $15 \mathrm{~min}$, a $25^{\circ} \mathrm{C}$, utilizando un extractor comercial casero, programado a alta velocidad, metodología propuesta por Chandegara et al. (2015). A partir de esta solución, se prepararon soluciones al 70, 80 y $90 \% \mathrm{v} / \mathrm{v}$ en agua destilada.

Almidón: Se utilizó almidón modificado de grado alimentario, con 98,8\% de pureza (Fécula Argo producida por Industria del maíz). Se prepararon soluciones de almidón al 0,5, 1 y 2\% p/v, en agua destilada, a $80^{\circ} \mathrm{C}$. Las soluciones, se agitaron a $3.000 \mathrm{rpm}$, por 20 min, con agitación magnética constante.

Aceite de Oliva: Se utilizó aceite de oliva extra virgen de marca comercial (Olivetto), obtenido de un mercado local del municipio de Palmira (Valle del Cauca, Colombia).

Aplicación de crioprotectores: Las hojas de lechuga desinfectadas, se sumergieron en las soluciones crioprotectoras por $2 \mathrm{~min}$, las cuales, se encontraban a $5 \pm 2^{\circ} \mathrm{C}$. El aceite de oliva, se aplicó con una brocha estéril, el exceso de aceite fue eliminado mediante centrifugación por $5 \mathrm{~min}$, utilizando una centrifuga casera de hortalizas.

Cinéticas de congelación: Se siguió la metodología propuesta por Ayala et al. (2010). Se construyeron curvas de temperatura vs tiempo; para ello, se utilizaron termocuplas tipo $\mathrm{K}$, adaptadas a un dataloguer (Modelo SDL200, USA) y un ultracongelador (New Bronswick, U.S.A.), a $-52^{\circ} \mathrm{C} \pm 3^{\circ} \mathrm{C}$. Durante una hora, con intervalos de $5 \mathrm{~s}$, se registraron las temperaturas de los diferentes tratamientos y se construyeron las curvas cinéticas. Como control, se congelaron hojas de lechuga sin crioprotección, en las mismas condiciones.

Para el análisis de las curvas de congelación, se siguió el modelo propuesto por Umaña (2011), quien divide la cinética de congelación en seis fases. Las fases, se identificaron de la siguiente manera: a) Etapa AS correspondió al subenfriamiento; b) Etapa SB, que indicó la liberación de calor latente tras la formación del primer cristal de hielo; c) Etapa BC, concentración de solutos; d) Etapa $\mathrm{CD}$, donde nuevamente se da liberación de calor latente; e) Etapa DE, que se refiere a la continuación de la cristalización de la mezcla y, finalmente, f) Etapa EF, que correspondió al descenso de la temperatura del material biológico hasta lograr el equilibrio del complejo de solutos y cristales de hielo.
Velocidad de congelación: Se determinó la velocidad de congelación de cada uno de los tratamientos, utilizando la ecuación 1, donde, Ti: Temperatura inicial del producto, $T_{\mathrm{f}}$ temperatura del producto congelado, $\Delta \mathrm{t}$ : Tiempo total del proceso.

$$
\theta=\mathrm{T}_{\mathrm{i}}-\mathrm{T}_{\mathrm{f}} /_{\Delta \mathrm{t}} \quad \text { Ecuación } 1
$$

Diseño de experimentos: Se utilizó un diseño de bloques con dos factores: factor 1, tipo de crioprotector con dos niveles (Aloe y almidón) y factor 2 concentración del crioprotector con tres niveles (concentración 1, 2 y 3). Adicionalmente, se realizó por triplicado, un tratamiento, en el cual, se utilizó aceite de oliva. El tratamiento control consistió en lechuga sin pretratamiento. Las variables dependientes fueron tiempo de duración, temperatura y velocidad de cada una de las diferentes etapas de congelación. Los datos, se analizaron mediante ANOVA, con una prueba de comparación Duncan. Se realizaron tres réplicas por cada tratamiento.

\section{RESULTADOS Y DISCUSIÓN}

Cinética de congelación de hojas de lechuga con crioprotector de Aloe: La figura 1 muestra las cinéticas de congelación de lechuga crioprotegida con Aloe y sus respectivas etapas. Se presentaron diferencias estadísticas entre las etapas de los tratamientos con Aloe y la muestra testigo (Figura 1a), a excepción de las etapas SB y BC (Tabla 1).

En la primera etapa, el tratamiento con concentración de $90 \%$ de Aloe, se presentó un tiempo de subenfriamiento mayor $(2,65 \mathrm{~min})$ al de la muestra testigo. En la etapa CD, las concentraciones de 80 y 90\% de Aloe tardaron más tiempo en la liberación de calor latente respecto a la muestra testigo y al tratamiento de Aloe al $70 \%$. Se hizo evidente que la diferencia de concentración de solutos influyó significativamente en el desarrollo de la etapa CD. En las etapas DE y EF, en los tratamientos Aloe al 80 y 90\%, se apreció reducción del tiempo, en el que se alcanzó el equilibrio entre solutos y cristales de hielo, con respecto al tratamiento testigo y al tratamiento Aloe al $70 \%$, tratamiento que tardaron más tiempo en alcanzar el equilibrio. Este último comportamiento, se debe a que en las etapas anteriores a DE el agua libre, presente en los tratamientos Aloe al 80 y 90\%, se congeló, dando lugar a que quedaran en la matriz extracelular solutos dispersos y en menor cantidad; sin embargo, es de aclarar que el agua libre en estos tratamientos es mínima, dado que la congelación rápida induce estados amorfos con formación mínima de agua congelable.

A pesar que el análisis estadístico entre los tratamientos con Aloe no mostró diferencias significativas en la etapa BC, se aprecia en esta etapa que el tratamiento al 90\% duró mayor tiempo (4,51 min) en formar mayor cantidad de cristales de hielo respecto a los demás tratamientos con Aloe y que la temperatura para alcanzar el punto $\mathrm{C}$ presentó un diferencial de, aproximadamente, $10^{\circ} \mathrm{C}$. Este comportamiento, se podría atribuir a la cantidad de solutos aportados por el gel al 90\%, los cuales, retardan la formación de 


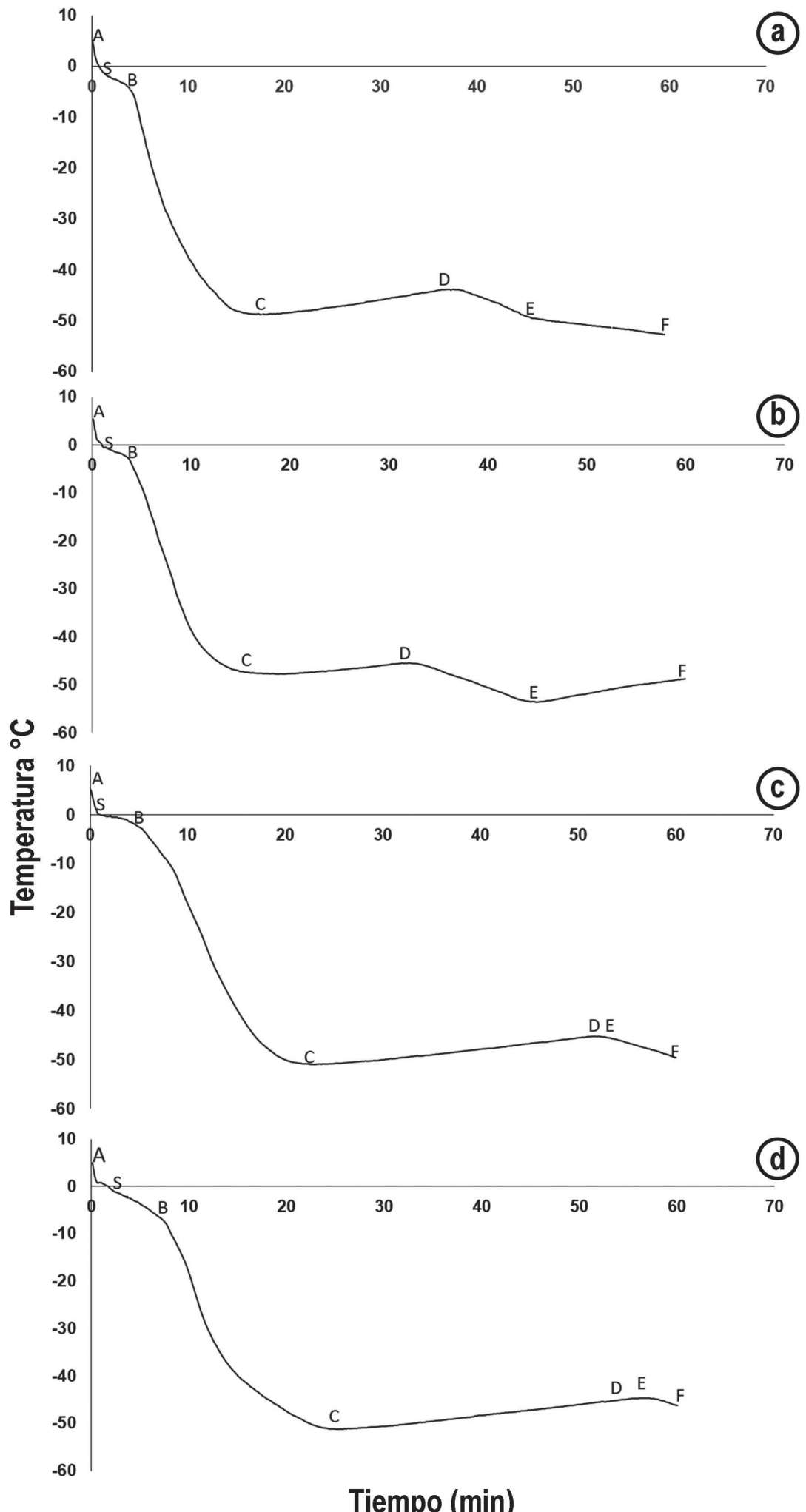

Figura 1. Cinéticas de congelación de Lactuca sativa L. "Lollo Bionda" crioprotegida con: a. Testigo sin crioprotector; b. Aloe 70\%; c. Aloe $80 \%$; d. Aloe 90\%. 
Tabla 1. Prueba del rango múltiple de DUNCAN para el tiempo de congelación en minutos de Lactuca sativa L. "Lollo Bionda", crioprotegida en las diferentes etapas de la curva de congelación.

\begin{tabular}{|c|c|c|c|c|c|c|}
\hline \multirow{4}{*}{ Tratamiento } & \multirow{3}{*}{ Concentración } & \multicolumn{5}{|c|}{ Tiempo de congelación (min) } \\
\hline & & \multicolumn{5}{|c|}{ Etapas de la curva de congelación } \\
\hline & & AS & SB & BC & $\mathrm{CD}$ & DE \\
\hline & Testigo & $1,59^{\mathrm{b}}$ & $1,15^{\mathrm{a}}$ & $3,94^{\mathrm{c}}$ & $4,49^{\mathrm{b}}$ & $3,37^{\mathrm{b}}$ \\
\hline \multirow{3}{*}{ Gel de Aloe } & $70 \%$ & $1,78^{\text {ba }}$ & $1,21^{\mathrm{a}}$ & $3,78^{\mathrm{c}}$ & $4,16^{\mathrm{b}}$ & $3,58^{\text {ba }}$ \\
\hline & $80 \%$ & $2,04^{\text {ba }}$ & $1,09^{\mathrm{a}}$ & $4,42^{\mathrm{bc}}$ & $5,49^{\mathrm{a}}$ & $1,69^{\mathrm{d}}$ \\
\hline & $90 \%$ & $2,65^{\mathrm{a}}$ & $1,11^{\mathrm{a}}$ & $4,51^{\mathrm{bc}}$ & $5,49^{\mathrm{a}}$ & $2,05^{\mathrm{cd}}$ \\
\hline \multirow{3}{*}{ Almidón } & $0,50 \%$ & $1,57^{\mathrm{b}}$ & $1,07^{\mathrm{a}}$ & $5,03^{\text {ba }}$ & $4,48^{\mathrm{b}}$ & $2,89^{\mathrm{bc}}$ \\
\hline & $1 \%$ & $2,10^{\text {ba }}$ & $1,14^{\mathrm{a}}$ & $5,38^{a}$ & $3,92^{\mathrm{b}}$ & $2,76^{\mathrm{bcd}}$ \\
\hline & $2 \%$ & $1,75^{\text {ba }}$ & $1,18^{\mathrm{a}}$ & $4,47^{\mathrm{bc}}$ & $4,50^{\mathrm{b}}$ & $2,08^{\text {cd }}$ \\
\hline Aceite de Oliva & $100 \%$ & $2,07^{\text {ba }}$ & $1,28^{\mathrm{a}}$ & $4,42^{\mathrm{bc}}$ & $1,78^{\mathrm{c}}$ & $4,55^{\mathrm{a}}$ \\
\hline
\end{tabular}

agua congelable, mientras que el tratamiento con concentración de $70 \%$, por tener menor cantidad de solutos, duró solo 3,78min.

Velocidad de congelación de lechuga con crioprotector de Aloe: Respecto a la velocidad con que se llevaron a cabo las etapas en la curva de congelación con crioprotector y sin crioprotector (Figura 1), el ANOVA no presentó diferencias significativas en las etapas $\mathrm{AS}, \mathrm{SB}, \mathrm{CD}$ y EF entre las diferentes concentraciones $(\mathrm{p}<0,05)$; no obstante, en las etapas BC y DE el análisis indicó diferencias entre tratamientos. La prueba Duncan indicó en la etapa $\mathrm{BC}$ p $<0,05$ entre los tratamientos con Aloe, al 70 y al $90 \%$ y, en los tratamientos con Aloe al $70 \%$, en la etapa DE.

Las soluciones de Aloe son hidrosolubles y de bajo peso molecular, por lo que son capaces de penetrar el tejido vegetal y cumplir un papel crioprotector. La crioprotección de sustancias penetrantes se atribuye, principalmente, a la habilidad que tiene este material de prevenir acumulación excesiva de electrolitos durante el proceso de congelación, a la formación de cristales de hielo pequeños dentro del citoplasma celular y a la modulación de la estabilidad de la bicapa de los fosfolípidos presente en la membrana eucariota de las células vegetales (Mesa Caro, 2011; Netzahualcóyotl, 2015).

No existen publicaciones científicas que involucren a Aloe vera como crioprotector; sin embargo, se podría citar la patente 2234431 (Martinez et al. 2005), en la que utilizaron Aloe vera al 99,9\%, por 5 a 30 min, como recubrimiento de frutas y de hortalizas, para prolongar su tiempo de vida comercial.

Cinética de congelación de lechuga con crioprotector de almidón: La figura 2 muestra las cinéticas de congelación de lechuga con crioprotector de almidón. El análisis estadístico con respecto al tiempo demostró diferencias significativas en la etapa BC $(p<0,05)$, que corresponde a la formación de los cristales de hielo (Tabla 1). La prueba Duncan indicó en la etapa BC que el tratamiento con almidón al 0,5 y 1\% son iguales a la muestra testigo (Figura 1a). Este comportamiento, se puede atribuir a que el almidón es viscoso y de alto peso molecular, por lo que no penetra la célula, lo que induce a que el crioprotector se deposite en los espacios intracelulares del tejido, por lo cual, la congelación del agua libre se da de forma rápida. Además, el comportamiento se explica porque los solutos con alto peso molecular, como el almidón, poseen altas temperaturas de transición vítrea $(\mathrm{Tg})$ y capacidad para formar mayor cantidad de agua congelable (Richards et al. 2002).

Velocidad de congelación de lechuga con crioprotector de almidón: EL ANOVA demostró que no hubo diferencia significativa entre las etapas AS, SB, CD y EF, en comparación con la muestra control (Tabla 2). Se presentaron diferencias significativas en la etapa $\mathrm{BC}$, entre la muestra testigo y el tratamiento con concentración de $2 \%$ de almidón $(\mathrm{p}<0,05)$. BC presentó mayor velocidad en el descenso de la temperatura $\left(2,08^{\circ} \mathrm{C} / \mathrm{min}\right)$ respecto al testigo; este comportamiento, se debe a la naturaleza no penetrante del almidón, que forma puentes hidrógeno con el agua, reduciendo la actividad de agua, a una magnitud mucho mayor que la que se predeciría por su concentración molar (Ávila-Portillo et al. 2006). Además, la mayor velocidad de descenso de la temperatura podría contribuir a la formación de menor cantidad de agua congelable y a que haya mayor cantidad de agua no congelable en la matriz amorfa o solución crioconcentrada (Umaña, 2011).

Como se indicó en la metodología, se utilizó agua a $80^{\circ} \mathrm{C}$, para preparar la solución crioprotectora de almidón, por lo cual, los componentes del almidón se encuentran como un sistema coloidal en estado sol y con una viscosidad ligeramente mayor. El almidón, como crioprotector, se caracteriza por no interactuar con el agua presente en el tejido vegetal y es incapaz de penetrar la pared celular por su alto peso molecular; sin embargo, protege externamente a las células vegetales. La crioprotección que ejercen estas dispersiones de almidón, se debe a que la adición del crioprotector genera estrés osmótico sobre las células vegetales de la lechuga, ya que aumenta la osmolaridad del medio; además, porque el almidón, a temperaturas de congelación, por debajo de la $\mathrm{Tg}$, inhibe la recristalización, debido a que es poco probable que la nucleación se lleve a cabo, 


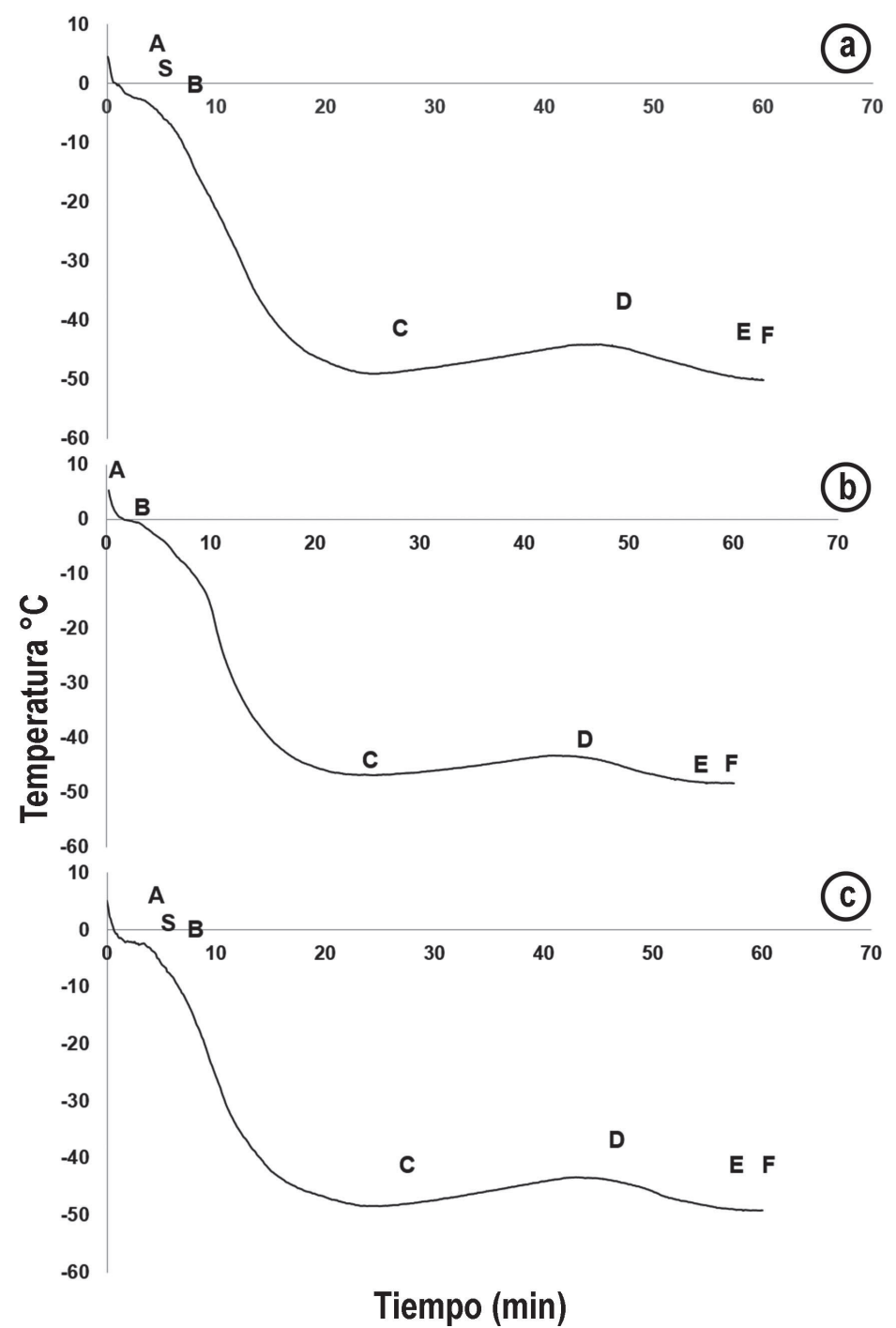

Figura 2. Cinéticas de congelación de Lactuca sativa L. "Lollo Bionda” crioprotegida con: a) Almidón 0,5\%; b) Almidón 1\% y c) Almidón 2\%.

Tabla 2. Prueba del rango múltiple de DUNCAN para la velocidad de congelación $\left({ }^{\circ} \mathrm{Cmin}^{-1}\right)$ de Lactuca sativa L. "Lollo Bionda", crioprotegida en las diferentes etapas de la curva de congelación).

\begin{tabular}{|c|c|c|c|c|c|c|c|}
\hline \multirow{4}{*}{ Tratamiento } & \multirow{3}{*}{ Concentración } & \multicolumn{6}{|c|}{ Velocidad de congelación $\left({ }^{\circ} \mathrm{Cmin}^{-1}\right)$} \\
\hline & & \multicolumn{6}{|c|}{ Etapas de la curva de congelación } \\
\hline & & AS & SB & BC & CD & $\mathrm{DE}$ & EF \\
\hline & Testigo & $2,73^{\mathrm{a}}$ & $3,21^{\mathrm{a}}$ & $2,08^{\text {ba }}$ & $1,10^{\mathrm{a}}$ & $1,16^{\text {ba }}$ & $1,14^{\text {bac }}$ \\
\hline \multirow{3}{*}{ Gel de Aloe } & $70 \%$ & $2,48^{a}$ & $3,21^{a}$ & $2,130^{\mathrm{a}}$ & $1,06^{\mathrm{a}}$ & $1,29^{\mathrm{a}}$ & $1,14^{\text {bac }}$ \\
\hline & $80 \%$ & $1,85^{\mathrm{a}}$ & $1,28^{a}$ & $1,93^{\mathrm{bac}}$ & $1,09^{\text {ba }}$ & $1,03^{\mathrm{b}}$ & $1,26^{\mathrm{a}}$ \\
\hline & $90 \%$ & $1,67^{\mathrm{a}}$ & $1,28^{a}$ & $1,87^{\mathrm{bc}}$ & $1,00^{\mathrm{a}}$ & $1,05^{\mathrm{b}}$ & $1,24^{\mathrm{ba}}$ \\
\hline \multirow{3}{*}{ Almidón } & $0,50 \%$ & $2,17^{\mathrm{a}}$ & $1,32^{\mathrm{a}}$ & $1,73^{\mathrm{dc}}$ & $1,12^{\mathrm{a}}$ & $1,35^{a}$ & $1,25^{\mathrm{a}}$ \\
\hline & $1 \%$ & $1,80^{\mathrm{a}}$ & $1,03^{\mathrm{a}}$ & $1,64^{\mathrm{d}}$ & $1,15^{\mathrm{a}}$ & $1,22^{\text {ba }}$ & $1,11^{\mathrm{bc}}$ \\
\hline & $2 \%$ & $2,45^{\mathrm{a}}$ & $2,14^{a}$ & $1,89^{\mathrm{bc}}$ & $1,13^{\mathrm{a}}$ & $1,03^{\mathrm{b}}$ & $1,19^{\mathrm{ba}}$ \\
\hline Aceite de Oliva & $100 \%$ & $2,01^{a}$ & $1,33^{\mathrm{a}}$ & $1,56^{\mathrm{d}}$ & $1,13^{\mathrm{a}}$ & $1,18^{\mathrm{ba}}$ & $1,03^{\mathrm{c}}$ \\
\hline
\end{tabular}


debido a la alta viscosidad del sistema y la dificultosa movilidad molecular (Gutiérrez et al. 2014). Las células vegetales, inicialmente, se deshidratan para compensar la fuerza osmótica inducida por la presencia de gránulos de almidón gelatinizado, que gelifica al enfriarse; sin embargo, el tejido se recupera. Además, al gelificar, el almidón actúa como una barrera a la difusión del agua intracelular. A esta deshidratación celular, se suma la deshidratación ocasionada por la ultracongelación, la cual, implica una rápida deshidratación celular, cuando se utiliza un crioprotector (Netzahualcóyotl, 2015). Por su parte, la amilosa del almidón, al ser un compuesto altamente soluble entra en contacto con el agua de los tejidos, aumentando la cantidad de sólidos solubles, alterando, así, las propiedades coligativas del producto. Los cambios en las concentraciones de agua intracelular se hacen evidentes en la curva, específicamente, en el punto $\mathrm{BC}$ de la congelación (Figura 2).

Cinética de congelación de lechuga con crioprotector de aceite de oliva: El ANOVA mostró diferencias significativas en la etapa
CD y DE $(p<0,05)$, con relación a la muestra testigo (Tabla 1$)$; la primera, corresponde a la liberación de calor latente, que se da por la sobresaturación, que alcanza uno de los solutos y su posterior cristalización, siendo evidente que el tiempo en el que se llevó a cabo esta etapa fue mayor en la muestra testigo (4,49min), en comparación con la muestra pretratada $(1,78 \mathrm{~min})$. Lo anterior, se atribuye a que el crioprotector aporta mayor cantidad de solutos en los espacios intercelulares y, principalmente, en los poros próximos a la superficie de la lechuga. Por último, el tiempo que se tardó en llegar al equilibrio (Etapa DE) fue mayor en la muestra crioprotegida (Figura 3).

Velocidad de congelación de lechuga con crioprotector de aceite de oliva: El ANOVA demostró diferencias significativas en las etapas $\mathrm{BC}$ de la muestra crioprotegida, en comparación con la muestra testigo; se observó, que la velocidad con que se llevó a cabo la etapa $\mathrm{BC}$ en la muestra testigo fue mayor que la del tratamiento con aceite de oliva, $2,08^{\circ} \mathrm{C} / \mathrm{min}$ y $1,56^{\circ} \mathrm{C} / \mathrm{min}$, respectivamente. Este comportamiento, se puede explicar, debido a que en la muestra

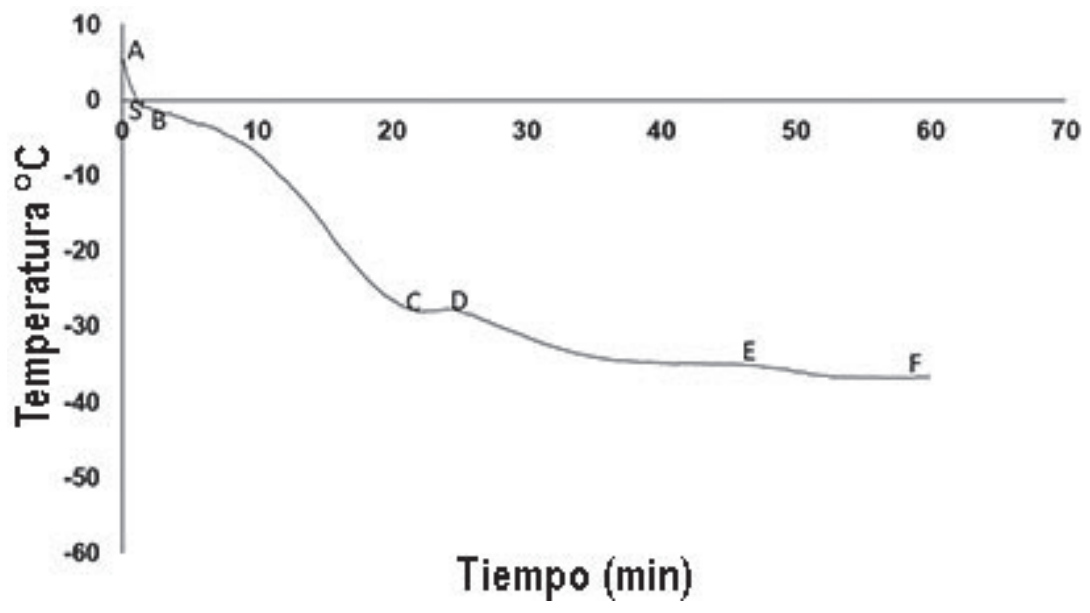

Figura 3. Cinética de congelación de Lactuca sativa L. "Lollo Bionda" crioprotegida con aceite de oliva.

testigo hay menor cantidad de sólidos solubles mientras que, en la muestra con aceite, hay mayor concentración de solutos en los espacios intra e intercelulares, lo cual, evita que los cristales de hielo se formen de manera rápida (Tabla 2).

Con referencia al aceite, el mecanismo de equilibrio del aceite-agua intracelular, se explica por qué el aceite de oliva desplaza el agua intracelular, a través de una difusión lenta que contrae, inicialmente, a la célula y luego la expande, evitando los daños producidos por la formación de cristales de hielo y, a su vez, manteniendo el volumen celular, impidiendo el colapso celular por excesiva deshidratación (Rota et al. 2006). El bajo peso molecular del aceite permite la entrada rápida, por medio de la membrana celular y afecta los procesos de solvatación de agua.

Comparando todos los tratamientos con la muestra patrón (Tabla 1), el ANOVA demostró diferencias significativas en los tiempos de todas las etapas a excepción de la etapa SB o punto de congelación $(\mathrm{p}<0,05)$; este comportamiento, se atribuyó a que el tipo de congelación fue rápida y los intervalos de tiempo en que fueron tomados los datos (5 s) no lograron permitir que se identificara esta etapa, por lo que no fue posible diferenciarla de manera óptima en los tratamientos. En la etapa AS, el tratamiento de Aloe al 90\%, tomó 5 min en llegar al subenfriamiento; este tiempo fue mayor comparado con los demás tratamientos y la mayor diferencia se presentó con la muestra testigo (1,59min). En la etapa CD, donde se da la liberación de calor por parte de los solutos que se sobresaturan, las soluciones de Aloe al 80 y 90\%, tardaron más tiempo en esta transición y ello se debe, a que tienen gran cantidad de azúcares y otros compuestos crioprotectores. Por otro lado, el ANOVA demostró que no hubo diferencias significativas en la velocidad de congelación para las etapas AS y SB; sin embargo, hubo diferencia para las demás etapas. La prueba de Duncan indicó que en la etapa BC, la muestra testigo, junto con la de Aloe al 70\%, tuvieron mayor velocidad de enfriamiento, lo que indica que en la etapa $\mathrm{BC}$ se presentó menor formación de cristales de hielo y mayor contenido 
de agua no congelable. El aceite de oliva presentó menor velocidad de congelación, debido a su naturaleza lipídica. La liberación de calor en la etapa CD se dio más rápido para las soluciones de almidón, mientras que en Aloe al 80 y 90\%, lentamente.

En las figuras 1, 2 y 3, se evidencia el aumento del tiempo de congelación con respecto a la muestra control, lo cual, según Machado \& Vélez (2008), se debe al incremento de sólidos solubles en la matriz sólida de lechuga. La cinética de congelación de L. sativa L. se midió solo en las hojas y no se incluyó el tallo, ni la base de las hojas. Según Umaña (2011), estas partes liberan humedad más lentamente que las hojas mismas; por lo tanto, los datos que se reportan en este artículo corresponden a hojas sin tallos. Valdívia et al. (2013) indican que el tiempo efectivo de congelación se refiere al tiempo requerido para reducir la temperatura del punto de enfriamiento desde la temperatura ambiente hasta $-15^{\circ} \mathrm{C}$; los autores, también afirman que cuanto mayor es la cantidad de solutos en una solución menor será la temperatura de inicio de la congelación, ya que se deprime el punto de congelación. La lechuga sin la aplicación de crioprotectores presenta menos sólidos solubles, por lo que la temperatura de inicio de congelación, es decir, el punto $\mathrm{SB}$, no se logra apreciar, como se indicó anteriormente, por ser una congelación rápida.

En esta investigación, se evidenció en un producto de alta sensibilidad a la congelación, como lo es la lechuga, la acción crioprotectora del aceite de oliva y de soluciones de diferentes concentraciones de Aloe vera y de almidón. Se concluyó que el tipo y la concentración del crioprotector modifica, de manera significativa, la temperatura, el tiempo y la velocidad de congelación de hojas de lechuga "Lollo Bionda". La concentración de los tratamientos influyó considerablemente en la acción crioprotectora, presentando mayor eficiencia los tratamientos de mayor concentración de solutos.

La adición de crioprotectores en el proceso de congelación resulta de interés para la conservación de la lechuga, en zonas donde no se cuenta con acceso al producto, de forma permanente.

Se sugiere desarrollar análisis microscópicos de la estructura del material biológico y, además, realizar análisis térmico por DSC, para identificar los cambios que sufre la lechuga en su estructura celular, tras aplicar los crioprotectores en el proceso de congelación.

Financiación: Esta investigación fue financiada por la Dirección de Investigación y Extensión de la Universidad Nacional de Colombia, sede Palmira. Conflicto de intereses: El manuscrito fue preparado y revisado por los autores, quienes declaramos que no existe conflicto de intereses que ponga en riesgo la validez de los resultados presentados.

\section{REFERENCIAS}

1. ÁVILA-PORTILLO, L.M.; MADERO, J.I.; LÓPEZ, C.; LEÓN, M.F.; ACOSTA, L.; GÓMEZ, C.; DELGADO, L.G.; GÓMEZ, C.; LOZANO, J.M.; REGUERO, M.T.
2006. Fundamentos de criopreservación. Rev. Colombiana de Obstetricia y Ginecología. 57(4):291-300.

2. AYALA A., A.; SERNA C., L.; MOSQUERA V., E. 2010. Liofilización de pitahaya amarilla (Selenicereus megalanthus). Vitae.17(2):121-127.

3. CHANDEGARA, V.K.; NANDASANA, J.N.; KUMPAVATAND, M.T.; VARSHNEY, A.K. 2015. Effect of temperature on gel extraction from Aloe vera leaves. Agric. Eng. Int. CIGR J. 17(1):207-212.

4. DOMÍNGUEZ, F. R.N.; ARZATE, V. I.; CHANONA, P. J.; WELTI, J.S.; ALVARADO, G. J.; CALDERÓN, D. G.; GARIBAY, F. V.; GUTIÉRREZ, L.G.F 2012. El gel de Aloe vera: estructura, composición química, procesamiento, actividad biológica e importancia en la industria farmacéutica y alimentaria. Rev. Mexicana de Ingeniería Química. 11(1):23-43.

5. ENGELMANN, F. 2011. Use of biotechnologies for the conservation of plant biodiversity. Vitro Cellular \& Developmental Biology - Plant. 47(1):5-16. htps://doi. org/10.1007/s11627-010-9327-2

6. ESPARZA, J.R.; NAVARRO, A.; KENDALL, P.; FORTIS, M.; PRECIADO, P.; MEZA, J.A. 2013. Acceptability of fresh cut leaf lettuce treated with ascorbic acid applied by hydrocooling. Rev. Mexicana de Ciencias Agrícolas. 4(5):767-778. https://doi.org/10.29312/remexca.v4i5.1174

7. FABBRI, S.; OLSEN, S.I.; OWSIANIAK, M. 2018. Improving environmental performance of post-harvest supply chains of fruits and vegetables in Europe: Potential contribution from ultrasonic humidification. J. Cleaner Production 182(1):16-26. https://doi.org/10.1016/j. jclepro.2018.01.157

8. GOKOGLU, N. 2019. Novel natural food preservatives and applications in seafood preservation: a review. J. Sci. Food Agric. 99:2068-2077. https://doi.org/10.1002/jsfa.9416

9. GÓMEZ-SÁNCHEZ, A.I.; CERÓN-CARRILLO, T.G.; RODRÍGUEZ-MARTÍNEZ, V.; VÁZQUEZ-AGUILAR, M.M. 2007. Aspectos tecnológicos de la congelación en alimentos. Temas Selectos de Ingeniería de Alimentos. 1:80-96.

10. GURRUCHAGA, H.; CIRIZA, J.; SAENZ DEL BURGO, L.; RODRÍGUEZ, J.; SANTOS, E.; PRÓSPER, F.; PEDRAZ, J. 2015. Cryopreservation of microencapsulated murine mesenchymal stem cells genetically engineered to secrete erythropoietin. Internal J. Pharmaceutics. 485(1-2):15-24. https://doi.org/10.1016/j.ijpharm.2015.02.047 
11. GURRUCHAGA, H.; SAENZ DEL BURGO, L.; HERNÁNDEZ, R.; ORIVE, G.; SELDEN, C.; FULLER, B.; PEDRAZ, J. 2018. Advances in the slow freezing cryopreservation of microencapsulated cells. J. Controlled Release. 281(1):119-13. https://doi.org/10.1016/j. jconrel.2018.05.016

12. GUTIÉRREZ, L.F.; ARIAS, S.; GARZÓN, D.; LÓPEZ, D.M.; OSORIO, A. 2014. Transición vítrea en alimentos: sistemas binarios agua-carbohidratos. Vector. 9:21-28.

\section{INSTITUTO COLOMBIANO DE NORMAS TÉCNICAS} Y CERTIFICACIÓN-ICONTEC. 1994. Norma Técnica Colombiana Frutas y Hortalizas Frescas. Lechuga. NTC 1064. Disponible desde Internet en: https:/ / es.scribd.com/ document/58308199/NTC-1064-Lechuga (con acceso 08/12/2016).

14. KUSHNARENKO, S.V.; ROMADANOVA, N.V.; BEKEBAYEVA, M.O.; MATAKOVA, G.N. 2015. Improving a cryopreservation regulation of apical meristems for the creation of a cryobank of potato cultivars and hybrids. Biotechnology Theory Pract 2:3541. https://doi.org/10.1016/j.plantsci.2004.07.022

15. MACHADO VELASCO, K.M.; VÉLEZ RUIZ, J.F. 2008. Estudio de propiedades físicas de alimentos mexicanos durante la congelación y el almacenamiento congelado. Rev. Mexicana Ingeniería Química. 7(1):41-54.

16. MARCO, A.; SERRANO, F. 2012. Crioconservacion: herramienta para la conservacion ex situ de material vegetal. Cuadernos de Biodiversidad. 38(1):9-12. https:// doi.org/10.14198/cdbio.2012.38.03

17. MARTINEZ, D.; SERRANO, M.; GARRIDO V.; CASTILLO, S. 2005. Aplicación de Aloe vera como recubrimiento sobre frutas y hortalizas. Patente ES 2234431 A1. Oficina Española de Patentes y Marcas.

18. MESA CARO, E.F. 2011. Crioconservación de semen ovino utilizando Aloe Vera como coadyuvante del producto comercial Triladyl en concentraciones del 10\% y 30\%. Tunja: Fundación Universitaria Juan de Castellanos, Facultad de Ciencias Agrarias. Disponible desde internet en: https://issuu.com/medicinaveterinariajdc/docs/ criopreservaci_n_de_semen_ovino_ut

19. MINISTERIO DE AGRICULTURA Y DESARROLLO RURAL-MADR. 2017. Balance del Sector hortofrutícola en 2017. Disponible desde Internet en: http://www. asohofrucol.com.co/imagenes/BALANCE_SECTOR_ HORTIFRUTICOLA_DICIEMBRE_2017.pdf (con acceso 25/06/2019).
20. NĂSTASE, G.; PEREZ, P.A.; SERBAN, A.; DOBROVICESCU, A.; STEFANESCU, M.F.; RUBINSKY, B. 2016. Advantages of isochoric freezing for food preservation: A preliminary analysis. Internal Communications in Heat and Mass Transfer. 78:95-100.

21. NETZAHUALCÓYOTL MATA, M.F. 2015. Efecto de tratamientos crioprotectores en la sobrevivencia y estabilidad genética de germoplasma de vainilla (vanilla insignis). Universidad Veracruzana, Facultad de Ciencias Químicas. Disponible desde internet en: https://cdigital.uv.mx/bitstream/ handle/123456789/46816/NezahualcoyotlMataMFabiana. pdf? sequence $=2 \&$ is Allowed $=y$

22. PÉREZ C., A.; BETANCOURT B., A.; DUQUE O., A.; LOBO R., E. 2016. Criopreservación y almacenamiento de Mycoplasma spp. Rev. Salud Animal. 38(2):105-111. https://doi.org/10.1016/j.icheatmasstransfer.2016.08.026

23. RICHARDS, A.B.; KRAKOWKA, S.; DEXTER, L.; SCHMID, H.; WOLTERBECK, A.; WAALKENS-BERENDSEN, D.; SHIGOYUKI, A.; KURIMOTO, M. 2002. Trehalose: a review of properties, history of use and human tolerance, and results of multiple safeties studies. Food and Chemical Toxicology. 40:871-898. https://doi.org/10.1016/S02786915(02)00011-X

24. ROMADANOVA, N.; KUSHNARENKO, S.; KARASHOLAKOVA, L. 2017. Development of a common PVS2 vitrification method for cryopreservation of several fruit and vegetable crops. In Vitro Cell. \& Dev. Biol.- Plant. 53:382-393. https://doi.org/10.1007/s11627017-9849-y

25. ROTA, A.; MILANI, C.; CABIANCA, G.; MARTINI, M. 2006. Comparison between glycerol and ethyleneglycol for dog semen cryopreservation. Theriogenology. 65:1848-1858. https://doi.org/10.1016/j.theriogenology.2005.10.015

26. UMAÑA, U.E. 2011. Conservación de alimentos por frío. Refrigeración/Congelamiento. Fiagro y fusades proinnova. (El Salvador). 234p.

27. UNITED STATES DEPARTMENT OF AGRICULTURE, USDA. 2016. National Agricultal Statistics Service. Disponible desde Internet en: https:/ / www.nass.usda.gov/ Statistics_by_Subject/result.php?CA67122E-5AF3-3058B89C-6D375960D1F8\&sector=CROPS\&group=VEGE TABLES\&comm=LETTUCE (con acceso 18/06/2018).

28. VALDÍVIA, R.; ALVAREZ, G.; ALBERTO, D.; RIOS, M. 2013. Curvas de congelación. Universidad Nacional Agraria la Molina. Lima, Perú: s.n. Investigación de pregrado. 